\title{
When nano becomes smart: Nanomaterial-containing medical devices
}

\section{Federica Scaletti* \\ Department of Chemistry, University of Massachusetts, Amherst, USA}

In the past year's nanomaterials came into use in healthcare, electronics, cosmetics and other areas. Nanomaterials are materials, with at least one nanoscale dimension (i.e., nano-objects), or structured on a nanoscale (nanostructured materials). Examples of nanomaterials are nanoparticles of different shape (e.g. spherical, rod-shaped, cubical, star-shaped etc..) and material (gold, silver, silica, polymeric, etc...), quantum dots (nanoparticles of semiconductive material), carbon nanotubes (single- or multi-walled), and graphene. These materials are appealing due to their unique properties (e.g. optical, structural, electronic, etc..) respect to their bulk counterparts (i.e., without a nanometric dimension). Therefore, these promising nanomaterials are seeking a considerable impact in the field of medical devices for diagnosis, treatment and prevention of diseases. Many nanomaterialcontaining medical devices are currently under development and some have already been launched on the market or under development.

There is a broad spectrum of medical applications for nanomaterialcontaining medical devices, from traditional medical equipment to sophisticated electronic devices. A well-known example relies on the use of silver nanoparticles as antimicrobial for wound care (e.g. Silverlon ${ }^{\mathrm{TM}}$ Antimicrobial Wound Dressings from Argentum Medical, USA) or medical textiles such as surgical masks for filtering viruses (e.g. NanoFense $\mathrm{T}^{\mathrm{TM}}$ by Applied Nanoscience, USA). Other examples are silicon or zirconium oxides nanoparticles embedded in a photopolymerizable resin matrix for dental restoration (i.e., Patent US 6194481 B1: Mechanically strong and transparent or translucent composites made using zirconium oxide nanoparticles), or nanoparticles of hydroxyapatite $\left(\left[\mathrm{Ca}_{5}\left(\mathrm{PO}_{4}\right)_{6}(\mathrm{OH})_{2}\right]\right)$ and calcium phosphate $\left(\left[\mathrm{Ca}_{3}\left(\mathrm{PO}_{4}\right)_{2}\right)\right.$ for bone and teeth filling (e.g. Nanogel ${ }^{\circledR}$ from Teknimed, France).

More sophisticated devices include cardiovascular and surgical devices, such as nano-electromechanical systems to assist surgeons, implantable or wearable medical devices for the diagnosis and for continuous monitoring, containing nano-electronic components (e.g. data storage modules) diagnostic tools and therapeutic actuating elements. An example of the latter is the sub-cutaneous device for monitoring the level of sugar in blood, measuring the fluorescence of carbon nanotubes in the presence of sugar.

Great attention has also been addressed to nanotechnologies for cancer treatment. Most of these novel technologies using nanoparticles (i.e., photothermal therapy of cancer), are still under clinical studies and need further investigations before their use in clinics. However, the use of nanoparticles for photothermal therapy of cancer seems to be really beneficial when combined with conventional treatments. In fact, these techniques are supposed to be able to destroy tumors without damaging healthy tissues. Some examples under clinical investigation are AuroLase ${ }^{\oplus}$ Therapy, using gold metal shell and silica core nanoparticles, and other plasmonic particles such as rod-shaped gold nanoparticles (i.e. gold nanorods), both able to absorb the near infrared light provided by a laser source and convert it in localized heat, able to destroy selectively cancer cells.

Great efforts have been done on the discovery of new and more efficient nanomaterial-containing medical devices; however, current problems for nanomedicine involve understanding the issues related to the toxicity and the environmental impact of these nanomaterials. Moreover, the use of nanomaterials in medicine is often limited by the stability and the reproducibility of these nanomaterials; in fact, few of these have been approved by FDA for medical purposes. Therefore, a deeper understanding of the toxicity of these materials as well as of their use for realization of complex medical devices is urgent.
Correspondence to: Federica Scaletti, Department of Chemistry, University of Massachusetts, Amherst, USA, Tel: +1 (413) 404-6649; E-mail: scaletti@umass.edu

Received: September 20, 2016; Accepted: October 04, 2016; Published: October 05, 2016 